\title{
Review
}

\section{Review of the REPRISE Trial: Tolvaptan in Later-Stage Autosomal Dominant Polycystic Kidney Disease}

Soroush Nomigolzar, BSc, RPSGT*; Himax Patel, BSc*;

"These authors contributed equally to this work

Ross University School of Medicine, Miramar, FL 33027, USA

\section{${ }^{*}$ Corresponding authors}

Soroush Nomigolzar, BSc, RPSGT

Ross University School of Medicine, Miramar, FL 33027, USA; Tel. (305)799-1630; E-mail: soroushnomigolzar@students.rossu.edu

Himax Patel, BSc

Ross University School of Medicine, Miramar, FL 33027, USA; Tel. 919-274-1038; E-mail: himaxpatel@students.rossu.edu

\section{Article Information}

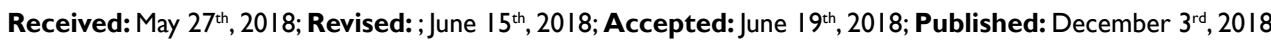

\section{Cite this article}

Nomigolzar S, Patel H, Review of the REPRISE trial:Tolvaptan in later-stage autosomal dominant polycystic kidney disease.J Nephrol. 20I8; 4(I): 7-I2. doi: $10.17140 / \mathrm{NPOJ}-4-119$

\section{ABSTRACT}

In the past, Tolvaptan Efficacy and Safety in Management of Autosomal Dominant Polycystic Kidney Disease (ADPKD) and its Outcomes (TEMPO) trial showed Tolvaptan, a vasopressin V2-receptor antagonist, slowed the decline in the estimated Glomerular Filtration Rate (eGFR) in patients with estimated creatinine clearance of $\geq 60 \mathrm{ml}$ per minute.

With this in consideration, Replicating Evidence of Preserved Renal Function: Investigation of Tolvaptan Safety and Efficacy (REPRISE) trial was conducted to assess the efficacy and safety of Tolvaptan in patients with later stage ADPKD. In this trial, 1370 patients with later-stage ADPKD were randomized. These patients were either 18 to 55 -years-old with eGFR of 25 to 65 $\mathrm{mL}$ per minute per $1.73 \mathrm{~m}^{2}$ of body surface area or 56 to 65 -years-old with eGFR of 25 to $44 \mathrm{~mL}$ per minute per $1.73 \mathrm{~m}^{2}$. The primary end-point of this trial was the change in eGFR between baseline and end of trial period, with adjustments made for exact duration that each patient participated for, interpolated to 1 year.

The change from baseline that was noted in the Tolvaptan group was $-2.34 \mathrm{~mL}$ per minute per $1.73 \mathrm{~m}^{2} v s .-3.61 \mathrm{~mL}$ per minute per $1.73 \mathrm{~m}^{2}$ in the placebo group. A difference of $1.27 \mathrm{~mL}$ per minute per $1.73 \mathrm{~m}^{2}(95 \% \mathrm{CI}, p<0.001)$ was noted.

Based on the data collected by the REPRISE trial over a 1-year period, it can be concluded that Tolvaptan slows the decline in eGFR than placebo in patients with later stage ADPKD.

\section{Keywords}

Tolvaptan Efficacy and Safety in Management of Autosomal Dominant Polycystic Kidney Disease and its Outcomes; ADPKD; REPRISE; TEMPO; Autosomal dominant polycystic kidney disease; Tolvaptan; Vasopressin V2-receptor antagonist

\section{Abbreviations}

ADPKD: Autosomal Dominant Polycystic Kidney Disease; REPRISE: Replicating Evidence of Preserved Renal function: Investigation of Tolvaptan Safety and Efficacy in ADPKD; TEMPO: Tolvaptan Efficacy and safety in Management of autosomal dominant Polycystic kidney disease and its Outcomes; eGFR: estimated Glomerular Filtration Rate; CKD: Chronic Kidney Disease; CI: Confidence Interval.
}

(a) Copyright 2018 by Nomigolzar S and Patel H. This is an open-access article distributed under Creative Commons Attribution 4.0 International License (CC BY 4.0), which allows to copy, redistribute, remix, transform, and reproduce in any medium or format, even commercially, provided the original work is properly cited. 


\section{INTRODUCTION}

utosomal Dominant Polycystic Kidney Disease (ADPKD) $\boldsymbol{A}_{\text {is one of the leading causes of end-stage kidney disease in }}$ adults. This disease results from mutations in the genes Polycystin-1 (PKD1) or Polycystin-2 (PKD2), with PKD1 located on short arm of chromosome 16 and PKD2 on the long arm of chromosome 4. ${ }^{1,2,4}$ It is also believed that in such patients, Vasopressin upon binding to V2 receptors, stimulates adenylyl cyclase to produce cAMP, causing cyst formation and fluid secretion. ${ }^{1}$ Therefore, V2receptor blockers such as Tolvaptan can reduce cyst formation and protect kidney function. ${ }^{1-3}$ In the TEMPO 3:4 trial, which involved patients with early ADPKD with3 creatinine clearance of $\geq 60$ $\mathrm{mL}$ per minute per $1.73 \mathrm{~m}^{2}$, Tolvaptan showed a reduced kidney proliferation and a slower decline in eGFR. ${ }^{5}$ In the TEMPO 4:4 trial, which was an open-label treatment which followed the same group of patients for additional two years, continued benefit of slower decline in eGFR was seen. ${ }^{6}$ However, in a small percent of the patient population, hepatotoxicity was noted. ${ }^{7}$ The patients in the TEMPO trials were monitored every four months, and two patients in the TEMPO 3:4 trial, and one patient in the TEMPO 4:4 trial suffered from a serious adverse drug-related liver injury. To prevent such adverse events and to increase safety of Tolvaptan in the patients of the REPRISE trial, monthly monitoring for toxic effects in the liver were conducted during the length of this trial. ${ }^{8}$

\section{METHODS}

The REPRISE trial was conducted from May 2014 to March 2016. Patients from 213 sites globally were enrolled in the trial. Eligible patients were either 18 to 55-years-old with eGFR of 25 to $65 \mathrm{~mL}$ per minute per $1.73 \mathrm{~m}^{2}$ or 56 to 65 -years-old with eGFR of 25 to $44 \mathrm{~mL}$ per minute per $1.73 \mathrm{~m}^{2}$. The older age group patients had a historical evidence of decline in eGFR of more than $2.0 \mathrm{~mL}$ per minute per $1.73 \mathrm{~m}^{2} .{ }^{9}$ Each enrolling site had their individual Institutional Review Board (IRB) review the protocol and the informed consent form (ICF). A group of investigators and representatives of the sponsor oversaw the trial. A safety monitoring committee was set up as well. At the end of the trial, all the data was collected and analyzed by the sponsor. The full article of the trial is available at NEJM.org. ${ }^{8}$

At the beginning of the trial, 2292 patients were eligible globally. Out of these 2292, $1519(66.2 \%)$ patients, entered an eight-week pre-randomization period that was divided into a screening phase, a single-blind placebo run-in phase, and a singleblind Tolvaptan phase, which comprised of a dose-adjustment phase and a run-in phase. At the end, 1370 patients $(58.7 \%)$ of the total eligible patients were randomized. During randomization, 685 patients (50\% of randomized) received placebo and 681 patients $(49.7 \%$ of randomized) received Tolvaptan (Figure 1).

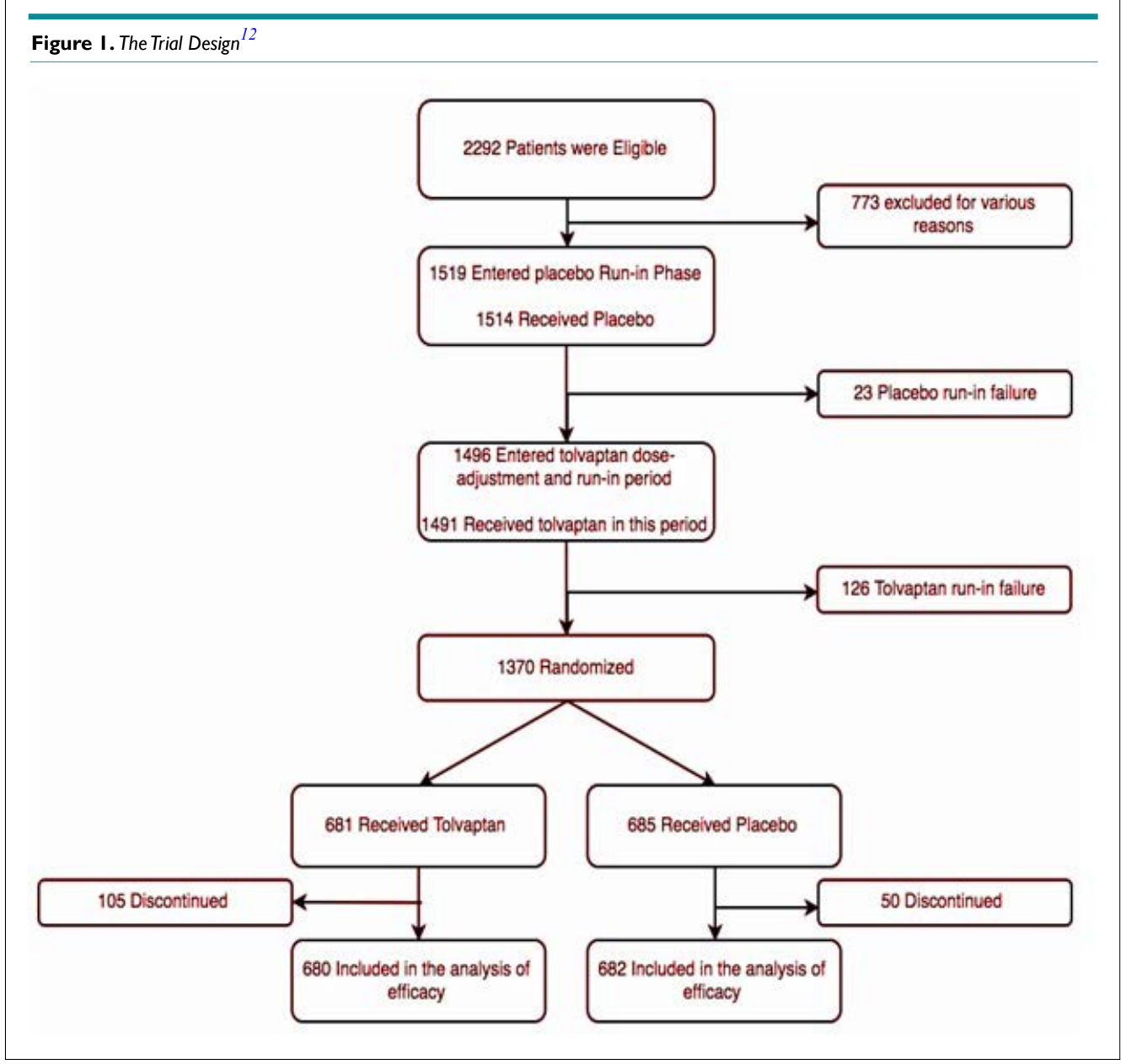


These patients were followed for a 1-year period, and at the end, they were required to have three follow-up lab visits between days 7 and 40 from receipt of last dose of assigned trial medication (Tolvaptan/placebo). Patients who were unable to complete the trial or withdrew early had their follow-up visits scheduled after the discontinuation of the trial medication with a final follow-up at the originally scheduled 12-month visit.

During this study, serum creatinine levels were measured centrally using mass spectroscopy. ${ }^{8,10}$ The eGFR calculations were based on the chronic kidney disease epidemiology collaboration equation. ${ }^{11}$

Patients were assessed and evaluated at the screening, placebo run-in, and single-blind Tolvaptan period, to establish a baseline.

Throughout the duration of the study, patients underwent monthly lab testing to evaluate for elevated ALT and elevated total bilirubin levels. Based on the reports of hepatotoxicity noted in patients during the TEMPO 3:4 trial, safety monitoring committee established the guideline for routine monthly monitoring. Elevations of ALT three times the upper normal limit of the normal range and elevations of total bilirubin more than two times the upper limit of normal range were reported.

\section{Primary End-Point}

The primary end-point of the study is the change in eGFR noted from the baseline (before placebo/Tolvaptan treatment) to followup at the end of the trial (after placebo/Tolvaptan treatment). Patients with early withdrawal had adjustments made with interpolation to 1-year. Without such adjustments, the group with more early withdrawals would be at an advantage, because patients with early withdrawal will have had less time for renal function decline, and such discrepancy would skew the data.

\section{Secondary End-Point}

The secondary end-point of the study is the slope of the change in eGFR, based on individual slopes of each patient. Similar interpolation to 1 -year was made in patients who withdrew early from the trial.

\section{RESULTS}

The statistical analysis of the primary end-point which was the mean $( \pm \mathrm{SE})$ change in the eGFR at 1 -year was $-2.34 \pm 0.24 \mathrm{~mL}$ per minute per $1.73 \mathrm{~m}^{2}$ (95\% CI, -2.81 to -1.87$)$ in the tolvaptan group, as compared with $-3.61 \pm 0.24 \mathrm{~mL}$ per minute per $1.73 \mathrm{~m}^{2}(95 \%$ CI, -4.08 to -3.14$)$ in the placebo group. The difference between eGFR decline in the tolvaptan group compared with the placebo group was $1.27 \mathrm{~mL}$ per minute per $1.73 \mathrm{~m}^{2}$ (95\% CI, 0.86 to 1.68; $p<0.001)$ indicating a significantly lower decrease in eGFR in the tolvaptan group. This benefit was also portrayed when comparing subgroups within the tolvaptan and placebo groups except in the older than 55-years of age, nonwhite and stage 2 chronic kidney disease categories where there was an absence of a statistically significant difference (Figure 2).

The key secondary end-point in this study was the mean slopes of the change in eGFR at 1-year which in the tolvaptan group was $-3.16 \pm 0.14 \mathrm{~mL}$ per minute per $1.73 \mathrm{~m}^{2}$ (95\% CI, -3.43 to -2.89$)$ as compared with $-4.17 \pm 0.14 \mathrm{~mL}$ per minute per 1.73

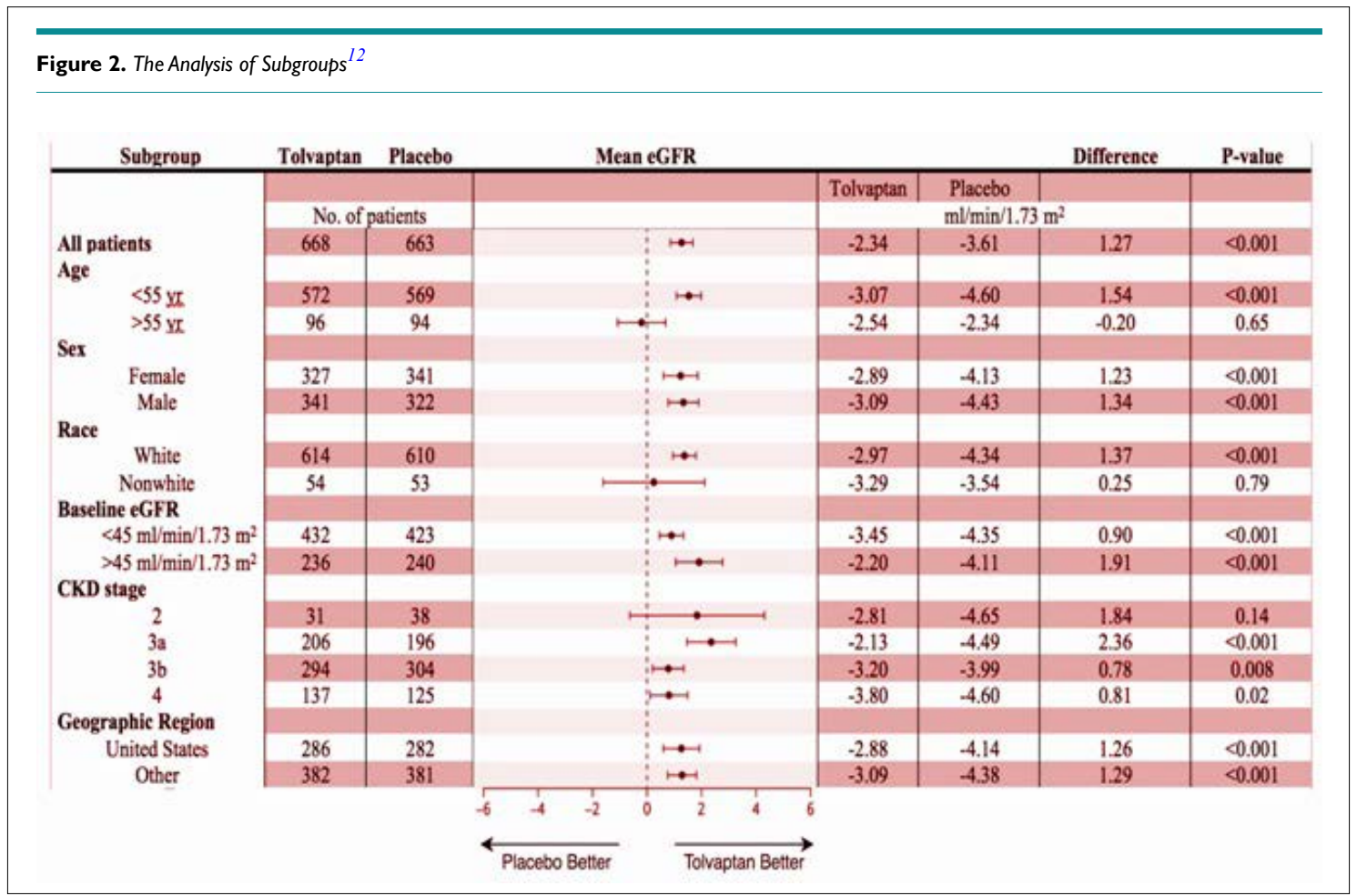


$\mathrm{m}^{2}(95 \%$ CI, -4.45 to -3.89$)$ in the placebo group. This category showed a statistically significant difference between the two groups which was $1.01 \mathrm{~mL}$ per minute per $1.73 \mathrm{~m}^{2}$ (95\% CI, -0.62 to -1.40; $p<0.001)$. Subgroup analysis in the secondary end-point showed a beneficial effect of tolvaptan in all the categories except those who were in the subgroups of older than 55-years of age, nonwhite and stage 2 chronic kidney disease all of which did not show a statistically significant difference (Figure 2).

The rates of adverse events in the 5-week single-blind tolvaptan period, were higher than the group administered with tolvaptan in the 1 -year double-blind period. During the doubleblind period, the rates of adverse events between the tolvaptan and placebo group did not have a notable difference and were $85.3 \%$ and $82.3 \%$ respectively. Patients in the tolvaptan group had higher rates of polyuria, nocturia, thirst, polydipsia, dry mouth, diarrhea, and fatigue, whereas those in the placebo group had higher rates of peripheral edema, kidney pain, and urinary tract infection. The adverse events patients experienced during this trial were mild to moderate in severity. In this study, 101 of 1491 patients $(6.8 \%)$ in the single-blind tolvaptan period, 65 of 681 patients $(9.5 \%)$ receiving tolvaptan in the double-blind period, and 15 of 685 $(2.2 \%)$ receiving placebo in the double-blind period, experienced significant adverse events which resulted in discontinuation of trial regimen in these individuals.

Another variable that was compared between the two groups was the liver enzymes. The alanine aminotransferase levels exceeded three times the upper limit of the normal range in 38 patients $(5.6 \%)$ in the tolvaptan group, as compared with $8(1.2 \%)$ in the placebo group. In either group there were no patients with bilirubin levels elevated to more than two times the upper limit of the normal range. The elevated liver-enzymes in all cases returned to normal following the withholding or discontinuation of the treatment.

\section{DISCUSSION}

Based on the analysis of the primary end-point, patients with more advanced ADPKD (mean [ $\pm \mathrm{SD}]$ eGFR of $41.0 \pm 11.1 \mathrm{~mL}$ per minute per $1.73 \mathrm{~m}^{2}$ at a mean $[ \pm \mathrm{SD}] 47 \pm 8$ years of age) who can tolerate tolvaptan without an unacceptable level of adverse effects, can benefit from a 1-year course of tolvaptan treatment as a result of lower decrease in eGFR compared to placebo which indicates a slower loss of renal function in these individuals. Moreover, the key secondary end-point depicted a difference between mean slopes of the change in eGFR of $1.01 \mathrm{~mL}$ per minute per 1.73 $\mathrm{m}^{2}$ in a year $(95 \% \mathrm{CI}, 0.62$ to $1.40 ; p<0.001)$ that was similar to $1.20 \mathrm{~mL}$ per minute per $1.73 \mathrm{~m}^{2}$ per year that was observed in the TEMPO 3:4 trial, which involved patients with early-stage ADPKD (mean $[ \pm \mathrm{SD}$ ] estimated GFR, 81.6 $\pm 29.6 \mathrm{~mL}$ per minute per $1.73 \mathrm{~m}^{2}$ at a mean $[ \pm \mathrm{SD}] 39 \pm 7$ years of age $)^{5}$. Furthermore, the eGFR benefit in the tolvaptan group in 3 years of TEMPO 3:4 trial $\left(3.34 \mathrm{~mL}\right.$ per minute per $\left.1.73 \mathrm{~m}^{2}, p<0.001\right)$ was preserved 2 years later in the TEMPO 4:4 open-label extension study $(3.15 \mathrm{~mL}$ per minute per $\left.1.73 \mathrm{~m}^{2}, p<0.001\right)$ where all the patients received tolvaptan treatment. ${ }^{13}$
The statistical analysis of the primary and secondary end-points portrayed the beneficial effect of tolvaptan in all the categories except those who were in the subgroups of older than 55 years of age, nonwhite and stage 2 chronic kidney disease (Figure 2). However, these subgroups contained a relatively smaller subject size compared to other subgroups and as a result, it cannot firmly be inferred that tolvaptan has no beneficial effect in these groups.

As a result of tolvaptan causing serious adverse drugrelated liver injury in some of the patients enrolled in TEMPO trials, the REPRISE trial monitored the patients' liver enzyme levels in a more frequent manner for toxic effects of tolvaptan. ${ }^{8}$ This system had the benefit of discontinuing the treatment following elevation in liver-enzymes which lead to return of enzymes to the normal range. Based on the results of this study, the liver enzyme elevations were temporary and there were no permanent liver damage; indicating that more frequent monitoring and a timely discontinuation of treatment is effective in preventing liver injury in patients being treated with tolvaptan.

The REPRISE study had some limitations that could be addressed in future studies. The main one being measurement of tolvaptan effect in patients with advanced stage ADPKD in a 1 -year period and as a result, the long-term benefits of tolvaptan in this patient population cannot be inferred based on these results.

However, the post hoc analysis of patients with stage $3 \mathrm{CKD}$ in the TEMPO 3:4 trial portrayed beneficial effects of tolvaptan over the 3 years of that study $(1.67 \mathrm{~mL}$ per minute per $1.73 \mathrm{~m}^{2}$ per year). ${ }^{14}$ Another limitation of this study is that it did not include the total kidney volume as was done in the TEMPO trial. This measurement would have enriched the data and helped gain a better understanding of Tolvaptan effect on kidney size. Finally, the patients in the REPRISE trial were asked to maintain good hydration which leads to vasopressin release suppression in the placebo group patients. This limits the outcome of the trial by underestimating the benefit of tolvaptan treatment.

In conclusion, Tolvaptan was shown to slow the progressive loss of renal function in patients with advanced ADPKD in a 1-year course of treatment. Future studies must be performed in order to further investigate the long-term benefit of Tolvaptan treatment in patients with later stage ADPKD following the 1 -year period. Also, future studies should aim to include a larger patient population in order to find out if there is indeed no benefit in using tolvaptan in patients from subgroups of older than 55 -years of age, nonwhite and stage 2 chronic kidney disease. Frequent monitoring of the liver-enzyme levels on a monthly basis, helped reduce drug-induced liver injury, possibly due to early detection and discontinuation of therapy.

Following the TEMPO trials' outcome, tolvaptan which is indicated to slow the decline in kidney function in adults at risk of rapidly progressing ADPKD was approved for use in ADPKD by regulatory agencies in Japan, Canada, and Western Europe. In 2013, the US Food and Drug Administration (FDA) requested 
a confirmatory trial, although as of 2018, tolvaptan has been approved for the use in ADPKD. ${ }^{15}$

The TEMPO trial and the REPRISE trial were not first of its kind in which scientists used a drug to study its effect on the progression of ADPKD. In the past, drugs such as Sirolimus (mTOR inhibitor) and Somatostatin analogues have been extensively studied to assess their therapeutic benefits versus risks in patients with ADPKD.

From September 2010 to March 2012, a prospective, single-center, randomized, open label study was conducted in which individuals were randomized to sirolimus added on to conventional treatment therapy OR conventional treatment alone, with the primary endpoint of eGFR change at 1-and 3-years compared to baseline. At the 1-year analysis, it was observed that the GFR fell from $26.7 \pm 5.8$ to $21.3 \pm 6.3 \mathrm{~mL}$ per minute per $1.73 \mathrm{~m}^{2}$ in the sirolimus group and $29.6 \pm 5.6$ to $24.9 \pm 6.2 \mathrm{~mL}$ per minute per 1.73 $\mathrm{m}^{2}$ in the conventional treatment group. ${ }^{16}$ Increased albuminuria and proteinuria were also observed in the group with sirolimus. Fourteen individuals in the sirolimus treatment group developed upper respiratory tract infections versus 11 in the control group. Three patients on sirolimus had angioedema, 14 had aphthous stomatitis, and seven patients had acne. At one year, total kidney volume increased by $9.0 \%$ in the sirolimus group versus $4.3 \%$ in the conventional therapy group. ${ }^{16}$ Therefore it was concluded, that mTOR inhibitor therapy was contraindicated and ineffective in patients with ADPKD.

A similar trial was conducted observing the effect of somatostatin analogue, octreotide, and its effect on ADPKD. From April 2006 to May 2008, a randomized, single-blind, placebo controlled trial was conducted in which individuals were randomized to a 3-year treatment with two $20 \mathrm{mg}$ IM octreotideLAR (long-acting release) or $0.9 \%$ sodium chloride solution every 28 days, with primary endpoint of total kidney volume (TKV) change at 1-year and 3-year follow-up. At 1-year follow-up, it was noted that the mean increase in the TKV was much slower in the octreotide group $(46.2 \mathrm{~mL})$ compared to the placebo group $(143.7 \mathrm{~mL}) .{ }^{17}$ At the 3 -year follow-up, mean increase from baseline in octreotide group was $220.1 \mathrm{~mL}$ compared to $454.3 \mathrm{~mL} .^{17}$ There was no difference in the number of adverse events seen in both groups. Based on the findings provided by this study, it was concluded that somatostatin analogues have protective effect against renal function loss in patients with ADPKD. ${ }^{17}$

Trials of this sort should encourage more studies on pharmacological agents in order to better understand their therapeutic benefits versus risks in patients with ADPKD. And, if they are proven to be therapeutically beneficial with minimal adverse effects, they need to be used in treating our patient population with ADPKD to slow their kidney function decline.

\section{CONFLICTS OF INTEREST |}

The authors declare that they have no conflicts of interest.

\section{REFERENCES}

1. Halvorson CR, Bremmer MS, Jacobs SC. Polycystic kidney disease: Inheritance, pathophysiology, prognosis, and treatment. Int J Nephrol Renovasc Dis. 2010; 3: 69-83. doi: https://doi.org/10.2147/ IJNRD.S6939

2. Patel V, Chowdhury R, Igarashi P. Advances in the pathogenesis and treatment of polycystic kidney disease. Curr Opin Nephrol Hypertens. 2009; 18(2): 99-106. doi: 10.1097/MNH.0b013e3283262ab0

3. Gattone II VH, Wang X, Harris PC, Torres VE. Inhibition of renal cystic disease development and progression by a vasopressin V2 receptor antagonist. Nat Med. 2003; 9(10): 1323-1326. doi: $10.1038 / \mathrm{nm} 935$

4. Chapman AB. Approaches to testing new treatments in autosomal dominant polycystic kidney disease: insights from the CRISP and HALT-PKD studies. Clin J Am Soc Nephrol. 2008; 3(4): 1197-1204. doi: 10.2215/CJN.00060108

5. Torres VE, Chapman AB, Devuyst O, et.al Tolvaptan in patients with autosomal dominant polycystic kidney disease. $N$ Engl J Med. 2012; 367(25): 2407-2418. doi: 10.1056/NEJMoa1205511

6. Torres VE, Chapman AB, Devuyst O, et al. Multicenter, openlabel, extension trial to evaluate the long-term efficacy and safety of early versus delayed treatment with tolvaptan in autosomal dominant polycystic kidney disease: the TEMPO 4:4 Trial. Nephrol Dial Transplant. 2017; 32(7): 1262. doi: 10.1093/ndt/gfx079

7. Watkins PB, Lewis JH, Kaplowitz N, et al. Clinical pattern of tolvaptan-associated liver injury in subjects with autosomal dominant polycystic kidney disease: Analysis of clinical trials database. Drug Saf. 2015; 38(11): 1103-1113. doi: 10.1007/s40264015-0327-3

8. Torres VE, Devuyst O, Chapman AB, et al. REPRISE Trial Investigators. Rationale and design of a clinical trial investigating tolvaptan safety and efficacyin autosomal dominantpolycystickidney disease. Am JNephrol.2017;45(3):257-266. doi: 10.1159/000456087

9. Pei Y, Obaji J, Dupuis A, et al. Unified criteria for ultrasonographic diagnosis of ADPKD. J Am Soc Nephrol. 2009; 20(1): 205-212. doi: 10.1681/ASN.2008050507

10. Levey AS, Coresh J, Greene T, et al. Using standardized serum creatinine values in the modification of diet in renal disease study equation for estimating glomerular filtration rate. Ann Intern Med. 2006; 145(4): 247-254.

11. Levey AS, Stevens LA, Schmid CH, et al. A new equation to estimate glomerular filtration rate. Ann Intern Med. 2009; 150(9): 604-612. doi: 10.7326/0003-4819-150-9-200905050-00006

12. Torres VE, Chapman AB, Devuyst O, et al. Tolvaptan in later- 
stage autosomal dominant polycystic kidney disease. $N$ Engl J Med. 2017; 377(20): 1930-1942. doi: 10.1056/NEJMoa1710030

13. Torres VE, Chapman AB, Devuyst O, et al. Multicenter, openlabel, extension trial to evaluate the long-term efficacy and safety of early versus delayed treatment with tolvaptan in autosomal dominant polycystic kidney disease: The TEMPO 4:4 Trial. Nephrol Dial Transplant. 2017; 32: 1262-1262. doi: 10.1093/ndt/gfx079

14. Torres VE, Higashihara E, Devuyst O, et al. Effect of tolvaptan in autosomal dominant polycystic kidney disease by CKD stage: Results from the TEMPO 3:4 Trial. Clin J Am SocNephrol. 2016; 11(5): 803-811. doi: 10.2215/CJN.06300615

15. U. S. Food and Drug Administration/Department of Health and Human Services. New Drug Application Approval. 2018. Web site: https://www.accessdata.fda.gov/drugsatfda_docs/appletter/ 2018/204441Orig1s0001tr.pdf. Accessed May 26, 2018

16. Ruggenenti P, Gentile G, Perico N, et al. Effect of sirolimus on disease progression in patients with autosomal dominant polycystic kidney disease and CKD stages 3b-4. Clin J Am SocNephrol. 2016; 11(5): 785-794. doi: 10.2215/CJN.09900915

17. Caroli A, Perico N, Perna A, et al. Effect of longacting somatostatin analogue on kidney and cyst growth in autosomal dominant polycystic kidney disease (ALADIN): A randomised, placebo-controlled, Multicentre trial. Lancet. 2013; 382(9903): 1485-1495. doi: 10.1016/S0140-6736(13)61407-5 